\title{
OPERATORS ON CONTROLLED $K$-G-FRAMES IN HILBERT SPACES
}

\author{
MARYAM FORUGHI, ELNAZ OSGOOEI, ASGHAR RAHIMI, \\ AND MOJGAN JAVAHERNIA
}

\begin{abstract}
Controlled frames for spherical wavelets were introduced by Bogdanova et al. to get a numerically more efficient approximation algorithm. In this paper we propose some useful results about controlled $K$-g-frames, which are generalizations of $K$-g-frames. We show that if $\left\{\Lambda_{i}\right\}_{i \in \mathbb{I}}$ is a $C C^{\prime}$-controlled $K$-g-frame and $U, K$ are bounded linear operators in a separable Hilbert space, then, under certain conditions, the sequences of operators $\left\{\Lambda_{i} U\right\}_{i \in \mathbb{I}},\left\{\Lambda_{i} U^{*}\right\}_{i \in \mathbb{I}}$ are also $C C^{\prime}$-controlled $K$-gframes. We also present the concept of $C C^{\prime}$-controlled $K$-g-dual frame and extend some known equalities and inequalities. Finally we study the stability problem for perturbation of $C C^{\prime}$-controlled $K$-g-frames.
\end{abstract}

\section{Introduction}

Frames which are generalization of orthonormal bases, were first introduced in the context of non-harmonic Fourier series by Duffin and Schaeffer in [7]. They provide robust, stable and usually non-unique representations of vectors in a Hilbert space. Theory of frames began to be more widely and deeply studied during the last 20 years with several new applications, e. g. signal processing, image processing, data compression, sampling theory and quantum computing. G-frames have been introduced by Sun in [18]. They are generalized frames and include ordinary frames and many recent generalizations of them, e. g., bounded quasi-projectors and fusion frames. Najati and et al. completed the concept of g-frames in [13] and proved some new results.

Frames for operators or $K$-frames have been introduced by Găvruţa in [9] to study the nature of atomic systems for a Hilbert space with respect to a bounded linear operator $K$. It is a well-known fact that $K$-frames are more general than the classical frames and due to higher generality, many properties of frames (such as invertibility of the frame operator, etc.) may not hold for $K$-frames.

Controlled frames for spherical wavelets are introduced in [2] to get a numerically more efficient approximation algorithm and the related theories are developed in $[1,15,16]$. The concept of controlled $g$-frames is presented in $[11,17]$ and also controlled $K$-frames are presented in [14]. Hua and Huang [10] introduced the concept of controlled $K$-g-frames.

2010 Mathematics Subject Classification. 42C15, 46C99, 41A58.

Key words and phrases. Controlled g-frame, controlled $K$-g-frame, controlled frame operator. 
In this paper, inspired by the results of Hua and Huang [10], we obtain some new results about $C C^{\prime}$-controlled $K$-g-frames.

Throughout the paper, $H$ is a separable Hilbert space, $\mathcal{B}(H)$ is the set of all bounded linear operators from $H$ into $H, \mathcal{G L}(H)$ is the set of all bounded linear operators which have bounded inverses and $\mathcal{G} \mathcal{L}^{+}(H)$ is the set of all positive operators in $\mathcal{G L}(H)$. The operators $C, C^{\prime} \in \mathcal{G} \mathcal{L}^{+}(H), K \in \mathcal{B}(H)$ and $\Lambda:=\left\{\Lambda_{i} \in\right.$ $\left.\mathcal{B}\left(H, H_{i}\right)\right\}_{i \in \mathbb{I}}$ is a sequence of bounded operators. Here $\mathbb{I} \subset \mathbb{Z}$.

Let us recall basic definitions and notations of controlled $K$-g-frames.

Lemma 1.1. ([6]). Suppose that $H_{1}$ and $H_{2}$ are Hilbert spaces and $L_{1} \in \mathcal{B}\left(H_{1}, H\right)$, $L_{2} \in \mathcal{B}\left(H_{2}, H\right)$. Then the following assertions are equivalent:

(1) $\mathcal{R}\left(L_{1}\right) \subseteq \mathcal{R}\left(L_{2}\right)$;

(2) $L_{1} L_{1}^{*} \leq \lambda^{2} L_{2} L_{2}^{*}$ for some $\lambda>0$;

(3) There exists a mapping $U \in \mathcal{B}\left(H_{1}, H_{2}\right)$ such that $L_{1}=L_{2} U$.

Moreover, if above conditions are valid, then there exists a unique operator $U$ such that

(a) $\|U\|^{2}=\inf \left\{\alpha>0 \mid L_{1} L_{1}^{*} \leq \alpha L_{2} L_{2}^{*}\right\}$;

(b) $\operatorname{ker}\left(L_{1}\right)=\operatorname{ker}(U)$;

(c) $\mathcal{R}(U) \subseteq \overline{\mathcal{R}\left(L_{2}^{*}\right)}$.

If an operator $U$ has a closed range, then there exists a right-inverse operator $U^{\dagger}$ (pseudo-inverse of $U$ ) in the following sense.

Lemma 1.2. (see [4]). Let $U \in \mathcal{B}\left(H_{1}, H_{2}\right)$ be a bounded operator with closed range $\mathcal{R}(U)$. Then there exists a bounded operator $U^{\dagger} \in \mathcal{B}\left(H_{2}, H_{1}\right)$ for which

$$
U U^{\dagger} x=x, \quad x \in \mathcal{R}(U) .
$$

Definition 1.1. [18] A family $\Lambda:=\left\{\Lambda_{i} \in \mathcal{B}\left(H, H_{i}\right)\right\}_{i \in \mathbb{I}}$ is called a g-frame for $H$ with respect to $\left\{H_{i}\right\}_{i \in \mathbb{I}}$, if there exist constants $0<A \leq B<\infty$ such that for each $f \in H$,

$$
A\|f\|^{2} \leq \sum_{i \in \mathbb{I}}\left\|\Lambda_{i} f\right\|^{2} \leq B\|f\|^{2} .
$$

For this frame, the g-frame operator is defined by

$$
S_{\Lambda}(f)=\sum_{i \in \mathbb{I}} \Lambda_{i}^{*} \Lambda_{i} f, \quad f \in H,
$$

which is positive and invertible.

Definition 1.2. [1] Let $C \in \mathcal{G L}(H)$. We say that $F:=\left\{f_{i}\right\}_{i \in \mathbb{I}}$ is a $C$-controlled frame for $H$ if there exist constants $0<A_{C} \leq B_{C}<\infty$ such that for each $f \in H$

$$
A_{C}\|f\|^{2} \leq \sum_{i \in \mathbb{I}}\left\langle f, f_{i}\right\rangle\left\langle C f_{i}, f\right\rangle \leq B_{C}\|f\|^{2} .
$$

Definition 1.3. [10] Let $C, C^{\prime} \in \mathcal{G L}^{+}(H)$ and $K \in \mathcal{B}(H)$. We say that $\Lambda:=$ $\left\{\Lambda_{i} \in \mathcal{B}\left(H, H_{i}\right)\right\}_{i \in \mathbb{I}}$ is a $\left(C, C^{\prime}\right)$-controlled $K$-g-frame for $H$ if there exist constants $0<A_{C C^{\prime}} \leq B_{C C^{\prime}}<\infty$ such that for each $f \in H$

$$
A_{C C^{\prime}}\left\|K^{*} f\right\|^{2} \leq \sum_{i \in \mathbb{I}}\left\langle\Lambda_{i} C^{\prime} f, \Lambda_{i} C f\right\rangle \leq B_{C C^{\prime}}\|f\|^{2} .
$$


If the right hand of (1.2) holds, $\Lambda$ is called a $\left(C, C^{\prime}\right)$-controlled $K$-g-Bessel sequence for $H$ with bound $B_{C}$. We call $\Lambda$ a Parseval $\left(C, C^{\prime}\right)$-controlled $K$-g-frame if

$$
\sum_{i \in \mathbb{I}}\left\langle\Lambda_{i} C^{\prime} f, \Lambda_{i} C f\right\rangle=\left\|K^{*} f\right\|^{2} .
$$

If $K=I_{H}$ then $\Lambda$ is called a $\left(C, C^{\prime}\right)$-controlled g-frame.

For simplicity, we will use a notation $C C^{\prime}$ instead of $\left(C, C^{\prime}\right)$.

If $\Lambda$ is a $C C^{\prime}$-controlled g-frame for $H$ and $C^{*} \Lambda_{i}^{*} \Lambda_{i} C^{\prime}$ is positive for all $i \in \mathbb{I}$, then for each $f \in H$

$$
A_{C C^{\prime}}\|f\|^{2} \leq \sum_{i \in \mathbb{I}}\left\|\left(C^{*} \Lambda_{i}^{*} \Lambda_{i} C^{\prime}\right)^{\frac{1}{2}} f\right\|^{2} \leq B_{C C^{\prime}}\|f\|^{2} .
$$

Now, let

$$
\mathfrak{K}_{2}:=\left\{\left(C^{*} \Lambda_{i}^{*} \Lambda_{i} C^{\prime}\right)^{\frac{1}{2}} f: f \in H\right\}_{i \in \mathbb{I}} \subset\left(\sum_{i \in \mathbb{I}} \oplus H\right)_{\ell^{2}} .
$$

It is easy to check that $\mathfrak{K}_{2}$ is a closed subspace of $\left(\sum_{i \in \mathbb{I}} \oplus H\right)_{\ell^{2}}$.

Now, we can define the synthesis and analysis operators of the $C C^{\prime}$-controlled g-frames as

$$
\begin{aligned}
T_{C C^{\prime}}: \mathfrak{K}_{2} & \longrightarrow H, \\
T_{C C^{\prime}}\left(\left\{\left(C^{*} \Lambda_{i}^{*} \Lambda_{i} C^{\prime}\right)^{\frac{1}{2}} f\right\}_{i \in \mathbb{I}}\right) & =\sum_{i \in \mathbb{I}} C^{*} \Lambda_{i}^{*} \Lambda_{i} C^{\prime} f
\end{aligned}
$$

and

$$
\begin{gathered}
T_{C C^{\prime}}^{*}: H \longrightarrow \mathfrak{K}_{2}, \\
T_{C C^{\prime}}^{*}(f)=\left\{\left(C^{*} \Lambda_{i}^{*} \Lambda_{i} C^{\prime}\right)^{\frac{1}{2}} f\right\}_{i \in \mathbb{I}} .
\end{gathered}
$$

Thus, the $C C^{\prime}$-controlled g-frame operator is given by

$$
S_{C C^{\prime}} f=T_{C C^{\prime}} T_{C C^{\prime}}^{*} f=\sum_{i \in \mathbb{I}} C^{*} \Lambda_{i}^{*} \Lambda_{i} C^{\prime} f, \quad f \in H .
$$

So,

$$
\left\langle S_{C C^{\prime}} f, f\right\rangle=\sum_{i \in \mathbb{I}}\left\langle\Lambda_{i} C^{\prime} f, \Lambda_{i} C f\right\rangle, \quad f \in H
$$

and

$$
A_{C C^{\prime}} I d_{H} \leq S_{C C^{\prime}} \leq B_{C C^{\prime}} I d_{H} .
$$

Lemma 1.3. [8] Let $C, C^{\prime} \in \mathcal{G L}^{+}(H)$. A sequence $\Lambda$ is a $C C^{\prime}$-controlled g-Bessel sequence for $H$ with bound $B_{C C^{\prime}}$ if and only if the operator

$$
\begin{aligned}
T_{C C^{\prime}}: \mathfrak{K}_{2} & \longrightarrow H, \\
T_{C C^{\prime}}\left(\left\{\left(C^{*} \Lambda_{i}^{*} \Lambda_{i} C^{\prime}\right)^{\frac{1}{2}} f\right\}_{i \in \mathbb{I}}\right) & =\sum_{i \in \mathbb{I}} C^{*} \Lambda_{i}^{*} \Lambda_{i} C^{\prime} f
\end{aligned}
$$

is well-defined and bounded with $\left\|T_{C C^{\prime}}\right\| \leq \sqrt{B_{C C^{\prime}}}$. 
Lemma 1.4. [8] Let $C, C^{\prime} \in \mathcal{G L}^{+}(H)$. A sequence $\Lambda$ is a $C C^{\prime}$-controlled g-frame for $H$ if and only if the operator

$$
\begin{aligned}
T_{C C^{\prime}}: \mathfrak{K}_{2} & \longrightarrow H, \\
T_{C C^{\prime}}\left(\left\{\left(C^{*} \Lambda_{i}^{*} \Lambda_{i} C^{\prime}\right)^{\frac{1}{2}} f\right\}_{i \in \mathbb{I}}\right) & =\sum_{i \in \mathbb{I}} C^{*} \Lambda_{i}^{*} \Lambda_{i} C^{\prime} f
\end{aligned}
$$

is well-defined, bounded and surjective.

Proposition 1.1. Let $\Lambda$ be a $C C^{\prime}$-controlled $K$-g-frame for $H$ and $K$ has a dense range. Suppose that $C^{*} \Lambda_{i}^{*} \Lambda_{i} C^{\prime}$ is positive and also $V_{i}:=\left(C^{*} \Lambda_{i}^{*} \Lambda_{i} C^{\prime}\right)^{\frac{1}{2}}$ for each $i \in \mathbb{I}$. Then $\left(\bigcap_{i \in \mathbb{I}} \operatorname{ker} V_{i}\right)^{\perp}=H$.

Proof. Assume that $A_{C C^{\prime}}$ and $B_{C C^{\prime}}$ are the frame bounds of $\Lambda$. Hence,

$$
A_{C C^{\prime}}\left\|K^{*} f\right\|^{2} \leq \sum_{i \in \mathbb{I}}\left\|\left(C^{*} \Lambda_{i}^{*} \Lambda_{i} C^{\prime}\right)^{\frac{1}{2}} f\right\|^{2} \leq B_{C C^{\prime}}\|f\|^{2} .
$$

Since ker $K^{*}=(\mathcal{R}(K))^{\perp}$ and $K$ has a dense range, $K^{*}$ is injective. Then from (1.3), for each $i \in I$, we get

$$
\bigcap_{i \in \mathbb{I}} \operatorname{ker} V_{i} \subseteq \operatorname{ker} K^{*}=\{0\} .
$$

So we get the proof.

Remark 1.1. Suppose that $\Lambda$ is a $C C^{\prime}$-controlled $K$-g-frame for $H$ with lower bound $A_{C C^{\prime}}$. Then we have $S_{C C^{\prime}} \geq A_{C C^{\prime}} K K^{*}$. So by Lemma 1.1, there exists an operator $U \in \mathcal{B}\left(H, \mathfrak{K}_{2}\right)$ such that

$$
T_{C C^{\prime}} U=K \text {. }
$$

Now, we can obtain optimal frame bounds of $\Lambda$ by the operator $U$. Indeed, it is obvious that

$$
B_{o p}=\left\|S_{C C^{\prime}}\right\|=\left\|T_{C C^{\prime}}\right\|^{2} .
$$

By Lemma 1.1, the equation (1.4) has a unique solution as $U_{0}$ such that

$$
\begin{aligned}
\left\|U_{0}\right\|^{2} & =\inf \left\{\alpha>0 \mid K K^{*} \leq \alpha T_{C C^{\prime}} T_{C C^{\prime}}^{*}\right\} \\
& =\inf \left\{\alpha>0 \mid\left\|K^{*} f\right\|^{2} \leq \alpha\left\|T_{C C^{\prime}}^{*} f\right\|^{2}, f \in H\right\} .
\end{aligned}
$$

Now, we have

$$
\begin{aligned}
A_{o p} & =\sup \left\{A>0 \mid A\left\|K^{*} f\right\|^{2} \leq\left\|T_{C C^{\prime}}^{*} f\right\|^{2}, f \in H\right\} \\
& =\left(\inf \left\{\alpha>0 \mid\left\|K^{*} f\right\|^{2} \leq \alpha\left\|T_{C C^{\prime}}^{*} f\right\|^{2}, f \in H\right\}\right)^{-1} \\
& =\left\|U_{0}\right\|^{-2} .
\end{aligned}
$$

\section{Operators preserving controlled $K$-g-frames}

In this section, for the $C C^{\prime}$-controlled $K$-g-frame $\left\{\Lambda_{i}\right\}_{i \in \mathbb{I}}$, we consider some proper relations between the operators $U, K \in B(H)$ and $C, C^{\prime} \in G L^{+}(H)$ and investigate the cases that $\left\{\Lambda_{i} U\right\}_{i \in \mathbb{I}},\left\{\Lambda_{i} U^{*}\right\}_{i \in \mathbb{I}}$ can also be $C C^{\prime}$-controlled $K$-gframes. Next, by putting connections between the operators $S_{\Lambda}, K, C$ and $C^{\prime}$, 
we reach to necessary and sufficient conditions that $\left\{\Lambda_{i}\right\}_{i \in \mathbb{I}}$ can be a Parseval $C C^{\prime}$-controlled $K$-g-frame.

Theorem 2.1. Let $\Lambda$ be a $C C^{\prime}$-controlled $K$-g-frame for $H$ and $U \in \mathcal{B}(H)$ such that $\mathcal{R}(U) \subset \mathcal{R}(K)$. Then $\Lambda$ is a $C C^{\prime}$-controlled $U$-g-frame for $H$.

Proof. Suppose that $A_{C C^{\prime}}$ is a lower frame bound of $\Lambda$. By Lemma 1.1, there exists $\alpha>0$ such that $U U^{*} \leq \alpha^{2} K K^{*}$. Now, for each $f \in H$ we have

$$
\frac{A_{C C^{\prime}}}{\alpha^{2}}\left\|U^{*} f\right\|^{2} \leq A_{C C^{\prime}}\left\|K^{*} f\right\|^{2} \leq \sum_{i \in \mathbb{I}}\left\langle\Lambda_{i} C^{\prime} f, \Lambda_{i} C f\right\rangle .
$$

So the proof is completed.

Theorem 2.2. Let $\Lambda$ be a $C C^{\prime}$-controlled $K$-g-frame for $H$. Assume that $K$ has a closed range and $U \in \mathcal{B}(H)$ such that $\mathcal{R}\left(U^{*}\right) \subseteq \mathcal{R}(K)$. Also suppose that $U^{*}$ commutes with $C$ and $C^{\prime}$. Then $\left\{\Lambda_{i} U^{*}\right\}_{i \in \mathbb{I}}$ is a $C C^{\prime}$-controlled $K$-g-frame for $\mathcal{R}(U)$ if and only if there exists $\delta>0$ such that for each $f \in \mathcal{R}(U)$,

$$
\left\|U^{*} f\right\| \geq \delta\left\|K^{*} f\right\| .
$$

Proof. Suppose that $\left\{\Lambda_{i} U^{*}\right\}_{i \in \mathbb{I}}$ is a $C C^{\prime}$-controlled $K$-g-frame for $H$ with a lower frame bound $E_{C C^{\prime}}$. If $B_{C C^{\prime}}$ is an upper frame bound of $\Lambda$, then for each $f \in \mathcal{R}(U)$

$$
E_{C C^{\prime}}\left\|K^{*} f\right\|^{2} \leq \sum_{i \in \mathbb{I}}\left\langle\Lambda_{i} U^{*} C^{\prime} f, \Lambda_{i} U^{*} C f\right\rangle \leq B_{C C^{\prime}}\left\|U^{*} f\right\|^{2} .
$$

Thus, $\left\|U^{*} f\right\| \geq \sqrt{\frac{E_{C C^{\prime}}}{B_{C C^{\prime}}}}\left\|K^{*} f\right\|$. For the opposite implication, for each $f \in H$ we have

$$
\left\|U^{*} f\right\|=\left\|\left(K^{\dagger}\right)^{*} K^{*} U^{*} f\right\| \leq\left\|K^{\dagger}\right\|\left\|K^{*} U^{*} f\right\| .
$$

Therefore, if $A_{C C^{\prime}}$ is a lower frame bound of $\Lambda$, we have

$$
\begin{aligned}
A_{C C^{\prime}} \delta^{2}\left\|K^{\dagger}\right\|^{-2}\left\|K^{*} f\right\|^{2} & \leq A_{C C^{\prime}}\left\|K^{\dagger}\right\|^{-2}\left\|U^{*} f\right\|^{2} \\
& \leq A_{C C^{\prime}}\left\|K^{*} U^{*} f\right\|^{2} \\
& \leq \sum_{i \in \mathbb{I}}\left\langle\Lambda_{i} U^{*} C^{\prime} f, \Lambda_{i} U^{*} C f\right\rangle .
\end{aligned}
$$

For the upper bound, it is clear that

$$
\sum_{i \in \mathbb{I}}\left\langle\Lambda_{i} U^{*} C^{\prime} f, \Lambda_{i} U^{*} C f\right\rangle \leq B_{C C^{\prime}}\|U\|^{2}\|f\|^{2} .
$$

So, $\left\{\Lambda_{i} U^{*}\right\}_{i \in \mathbb{I}}$ is a $C C^{\prime}$-controlled $K$-g-frame for $H$ with frame bounds $A_{C C^{\prime}} \delta^{2}\left\|K^{\dagger}\right\|^{-2}$ and $B_{C C^{\prime}}\|U\|^{2}$.

Theorem 2.3. Let $\Lambda$ be a $C C^{\prime}$-controlled $K$-g-frame for $H$ and the operator $K$ has a dense range. Assume that $U \in \mathcal{B}(H)$ has a closed range and $U$ and $U^{*}$ commute with $C$ and $C^{\prime}$. If $\left\{\Lambda_{i} U^{*}\right\}_{i \in \mathbb{I}}$ and $\left\{\Lambda_{i} U\right\}_{i \in \mathbb{I}}$ are $C C^{\prime}$-controlled $K$-gframe for $H$, then $U$ is invertible.

Proof. Assume that $\left\{\Lambda_{i} U^{*}\right\}_{i \in \mathbb{I}}$ is a $C C^{\prime}$-controlled $K$-g-frame for $H$ with frame bounds $A_{1}$ and $B_{1}$. Then for each $f \in H$

$$
A_{1}\left\|K^{*} f\right\|^{2} \leq \sum_{i \in \mathbb{I}}\left\langle\Lambda_{i} U^{*} C^{\prime} f, \Lambda_{i} U^{*} C f\right\rangle \leq B_{1}\|f\|^{2} .
$$


Since $K$ has a dense range, $K^{*}$ is injective. Then by $(2.1), U^{*}$ is injective. Moreover, $\mathcal{R}(U)=\left(\operatorname{ker} U^{*}\right)^{\perp}=H$. So $U$ is surjective.

Now, suppose that $\left\{\Lambda_{i} U\right\}_{i \in \mathbb{I}}$ is a $C C^{\prime}$-controlled $K$-g-frame for $H$ with frame bounds $A_{2}$ and $B_{2}$. Then, for each $f \in H$

$$
A_{2}\left\|K^{*} f\right\|^{2} \leq \sum_{i \in \mathbb{I}}\left\langle\Lambda_{i} U C^{\prime} f, \Lambda_{i} U C f\right\rangle \leq B_{2}\|f\|^{2} .
$$

Therefore $U$ is injective, since $\operatorname{ker} U \subseteq \operatorname{ker} K^{*}$. Thus, $U$ is an invertible operator and the proof is completed.

Theorem 2.4. Let $\Lambda$ be a $C C^{\prime}$-controlled $K$-g-frame for $H$ and $U \in \mathcal{B}(H)$ be a co-isometry (i.e. $U U^{*}=I d_{H}$ ) such that $U K=K U$ and $U^{*}$ commutes with $C$ and $C^{\prime}$. Then $\left\{\Lambda_{i} U^{*}\right\}_{i \in \mathbb{I}}$ is a $C C^{\prime}$-controlled $K$-g-frame for $H$.

Proof. Suppose that $\Lambda$ is a $C C^{\prime}$-controlled $K$-g-frame for $H$ with frame bounds $A_{C C^{\prime}}$ and $B_{C C^{\prime}}$. For each $f \in H$, we have

$$
\sum_{i \in \mathbb{I}}\left\langle\Lambda_{i} U^{*} C^{\prime} f, \Lambda_{i} U^{*} C f\right\rangle=\sum_{i \in \mathbb{I}}\left\langle\Lambda_{i} C^{\prime} U^{*} f, \Lambda_{i} C U^{*} f\right\rangle \leq B_{C C^{\prime}}\|U\|^{2}\|f\|^{2} .
$$

So, $\left\{\Lambda_{i} U^{*}\right\}_{i \in \mathbb{I}}$ is a controlled g-Bessel sequence. For the lower bound, we can write

$$
\begin{aligned}
\sum_{i \in \mathbb{I}}\left\langle\Lambda_{i} U^{*} C^{\prime} f, \Lambda_{i} U^{*} C f\right\rangle & =\sum_{i \in \mathbb{I}}\left\langle\Lambda_{i} C^{\prime} U^{*} f, \Lambda_{i} C U^{*} f\right\rangle \\
& \geq A_{C C^{\prime}}\left\|K^{*} U^{*} f\right\|^{2} \\
& =A_{C C^{\prime}}\left\|U^{*} K^{*} f\right\|^{2} \\
& =A_{C C^{\prime}}\left\|K^{*} f\right\|^{2} .
\end{aligned}
$$

Theorem 2.5. Let $\Lambda=\left\{\Lambda_{i} \in \mathcal{B}\left(H, H_{i}\right)\right\}_{i \in \mathbb{I}}$ and $\Theta:=\left\{\Theta_{i} \in \mathcal{B}\left(H, H_{i}\right)\right\}_{i \in \mathbb{I}}$ be two $C C^{\prime}$-controlled $K$-g-Bessel sequences for $H$ with bounds $B_{\Lambda}$ and $B_{\Theta}$, respectively. Suppose that $T_{\Lambda, C, C^{\prime}}$ and $T_{\Theta, C, C^{\prime}}$ are their synthesis operators such that $T_{\Theta, C, C^{\prime}} T_{\Lambda, C, C^{\prime}}^{*}=K^{*}$. Then $\Lambda$ and $\Theta$ are $C C^{\prime}$-controlled $K$ and $K^{*}$-g-frames, respectively.

Proof. For each $f \in H$ we have

$$
\begin{aligned}
\left\|K^{*} f\right\|^{4} & =\left\langle K^{*} f, K^{*} f\right\rangle^{2} \\
& =\left\langle T_{\Lambda, C, C^{\prime}}^{*} f, T_{\Theta, C, C^{\prime}}^{*} K^{*} f\right\rangle^{2} \\
& \leq\left\|T_{\Lambda, C, C^{\prime}}^{*} f\right\|^{2}\left\|T_{\Theta, C, C^{\prime}}^{*} K^{*} f\right\|^{2} \\
& =\left(\sum_{i \in \mathbb{I}}\left\langle\Lambda_{i} C^{\prime} f, \Lambda_{i} C f\right\rangle\right)\left(\sum_{i \in \mathbb{I}}\left\langle\Theta_{i} C^{\prime} K^{*} f, \Theta_{i} C K^{*} f\right\rangle\right) \\
& \leq\left(\sum_{i \in \mathbb{I}}\left\langle\Lambda_{i} C^{\prime} f, \Lambda_{i} C f\right\rangle\right) B_{\Theta}\left\|K^{*} f\right\|^{2}
\end{aligned}
$$

Thus

$$
B_{\Theta}^{-1}\left\|K^{*} f\right\|^{2} \leq \sum_{i \in \mathbb{I}}\left\langle\Lambda_{i} C^{\prime} f, \Lambda_{i} C f\right\rangle
$$


This means that $\Lambda$ is a $C C^{\prime}$-controlled $K$-g-frame for $H$. Similarly, since $T_{\Lambda, C, C^{\prime}} T_{\Theta, C, C^{\prime}}^{*}=$ $K, \Theta$ is a $C C^{\prime}$-controlled $K^{*}$-g-frame with lower bound $B_{\Lambda}^{-1}$.

Theorem 2.6. Let $\Lambda$ be a g-frame for $H$ with frame operator $S_{\Lambda}$. Also assume that $\Lambda$ is a $C C^{\prime}$-controlled $g$-Bessel sequence with frame operator $S_{C C^{\prime}}$. Then $\Lambda$ is a Parseval $C C^{\prime}$-controlled $K$-g-frame for $H$ if and only if $C=\left(S_{\Lambda}^{-p}\right)^{*} \Phi$ and $C^{\prime}=S_{\Lambda}^{-q} \Psi$ where $\Phi, \Psi$ are two operators on $H$ such that $\Phi^{*} \Psi=K K^{*}$ and $p+q=1$ where $p, q \in \mathbb{R}$.

Proof. Assume that $\Lambda$ is a Parseval $C C^{\prime}$-controlled $K$-g-frame for $H$. It is clear that $S_{C C^{\prime}}=C^{*} S_{\Lambda} C^{\prime}$ and $S_{C C^{\prime}}=K K^{*}$. Therefore, for each $p, q \in \mathbb{R}$ such that $p+q=1$, we obtain

$$
K K^{*}=C^{*} S_{\Lambda}^{p} S_{\Lambda}^{q} C^{\prime}
$$

We define $\Phi:=\left(S_{\Lambda}^{p}\right)^{*} C$ and $\Psi:=S_{\Lambda}^{q} C^{\prime}$. So

$$
\Phi^{*} \Psi=C^{*} S_{\Lambda}^{p} S_{\Lambda}^{q} C^{\prime}=K K^{*} .
$$

Conversely, Let $\Phi, \Psi$ be two operators on $H$ such that $\Phi^{*} \Psi=K K^{*}$. Suppose that $C=\left(S_{\Lambda}^{-p}\right)^{*} \Phi$ and $C^{\prime}=S_{\Lambda}^{-q} \Psi$ are two operators on $H$ where $p, q \in R$ and $p+q=1$. Since

$$
K K^{*}=\Phi^{*} \Psi=C^{*} S_{\Lambda}^{p} S_{\Lambda}^{q} C^{\prime}=C^{*} S_{\Lambda} C^{\prime}=S_{C C^{\prime}},
$$

so, for each $f \in H$

$$
\left\|K^{*} f\right\|^{2}=\left\langle K K^{*} f, f\right\rangle=\sum_{i \in \mathbb{I}}\left\langle C^{*} \Lambda_{i}^{*} \Lambda_{i} C^{\prime} f, f\right\rangle .
$$

Thus $\Lambda$ is a Parseval $C C^{\prime}$-controlled $K$-g-frame for $H$.

\section{Duals of controlled $K$-g-frames and some equalities}

In this section, by the concept of $K$-g-dual pair, we present a bounded operator called dual operator and propose some known equalities and inequalities between dual operator and $C C^{\prime}$-controlled $K$-g-frames.

Definition 3.1. Suppose that $\Lambda$ is a $C C^{\prime}$-controlled $K$-g-frame for $H$ with synthesis operator $T_{\Lambda, C, C^{\prime}}$. Then $\widetilde{\Lambda}:=\left\{\widetilde{\Lambda}_{i} \in \mathcal{B}\left(H, H_{i}\right)\right\}_{i \in \mathbb{I}}$ is called a $C C^{\prime}$-controlled $K$-g-dual frame (or brevity $C C^{\prime}$ - $K$-g-dual) for $\Lambda$ if

$$
T_{\Lambda, C, C^{\prime}} T_{\widetilde{\Lambda}, C, C^{\prime}}^{*}=K
$$

and $\widetilde{\Lambda}$ is a $C C^{\prime}$-controlled $K$-g-Bessel sequence.

In this case, $(\Lambda, \widetilde{\Lambda})$ is called a $C C^{\prime}$-controlled $K$-g-dual pair. The following result presents equivalent conditions of the $C C^{\prime}-K$-g-dual.

Proposition 3.1. Let $\widetilde{\Lambda}$ be a $C C^{\prime}-K$-g-dual for $\Lambda$. Then the following conditions are equivalent:

(1) $T_{\Lambda, C, C^{\prime}} T_{\widetilde{\Lambda}, C, C^{\prime}}^{*}=K$;

(2) $T_{\widetilde{\Lambda}, C, C^{\prime}} T_{\Lambda, C, C^{\prime}}^{*}=K^{*}$;

(3) for each $f, f^{\prime} \in H$, we have

$$
\left\langle K f, f^{\prime}\right\rangle=\left\langle T_{\widetilde{\Lambda}, C, C^{\prime}}^{*} f, T_{\Lambda, C, C^{\prime}}^{*} f^{\prime}\right\rangle .
$$


Proof. Straightforward.

Theorem 3.1. If $\widetilde{\Lambda}$ is a $C C^{\prime}-K$-g-dual for $\Lambda$, then $\widetilde{\Lambda}$ is a $C C^{\prime}$-controlled $K^{*}$-gframe for $H$.

Proof. Suppose that $f \in H$ and $B_{C}$ is an upper bound of $\Lambda$. Therefore,

$$
\begin{aligned}
\|K f\|^{4} & =|\langle K f, K f\rangle|^{2} \\
& =\left|\left\langle T_{\Lambda, C, C^{\prime}} T_{\widetilde{\Lambda}, C, C^{\prime}}^{*} f, K f\right\rangle\right|^{2} \\
& =\left|\left\langle T_{\widetilde{\Lambda}, C, C^{\prime}}^{*} f, T_{\Lambda, C, C^{\prime}}^{*} K f\right\rangle\right|^{2} \\
& \leq\left\|T_{\widetilde{\Lambda}, C, C^{\prime}}^{*} f\right\|^{2} B_{C}\|K f\|^{2} \\
& =B_{C}\|K f\|^{2} \sum_{i \in I}\left\langle\widetilde{\Lambda}_{i} C^{\prime} f, \widetilde{\Lambda}_{i} C f\right\rangle,
\end{aligned}
$$

and this completes the proof.

Corollary 3.1. Assume that $C_{o p}$ and $D_{o p}$ are the optimal bounds of $\widetilde{\Lambda}$, respectively. Then

$$
C_{o p} \geq B_{o p}^{-1} \quad \text { and } \quad D_{o p} \geq A_{o p}^{-1}
$$

for which $A_{o p}$ and $B_{o p}$ are the optimal bounds of $\Lambda$, respectively.

Assume that $(\Lambda, \widetilde{\Lambda})$ is a $C C^{\prime}$-controlled $K$-g-dual pair and $\mathbb{J} \subset \mathbb{I}$. We define

$$
S_{\sqrt{J}} f:=\sum_{i \in \mathbb{J}}\left(C^{*} \Lambda_{i}^{*} \Lambda_{i} C^{\prime}\right)^{\frac{1}{2}}\left(C^{*} \widetilde{\Lambda}_{i}^{*} \widetilde{\Lambda}_{i} C^{\prime}\right)^{\frac{1}{2}} f, f \in H,
$$

and we call it a dual operator.

It is easy to check that $S_{\sqrt{\rrbracket}} \in \mathcal{B}(H)$ and $S_{\mathbb{J}}+S_{\sqrt{ } c}=K$, where $\mathbb{J}^{c}$ is the complement of $\mathbb{J}$.

Now, by that operator $S_{\sqrt{ }}$ we extend some known equalities and inequalities for controlled $K$-g-frames in the following theorems.

Theorem 3.2. If $f \in H$ then

$$
\begin{aligned}
& \sum_{i \in \mathbb{J}}\left\langle\left(C^{*} \widetilde{\Lambda}_{i}^{*} \widetilde{\Lambda}_{i} C^{\prime}\right)^{\frac{1}{2}} f,\left(C^{*} \Lambda_{i}^{*} \Lambda_{i} C^{\prime}\right)^{\frac{1}{2}} K f\right\rangle-\left\|S_{J} f\right\|^{2} \\
= & \sum_{i \in J^{c}} \overline{\left\langle\left(C^{*} \widetilde{\Lambda}_{i}^{*} \widetilde{\Lambda}_{i} C^{\prime}\right)^{\frac{1}{2}} f,\left(C^{*} \Lambda_{i}^{*} \Lambda_{i} C^{\prime}\right)^{\frac{1}{2}} K f\right\rangle}-\left\|S_{J^{c}} f\right\|^{2}
\end{aligned}
$$

Proof. Let $f \in H$. We can write

$$
\begin{aligned}
& \sum_{i \in \mathbb{J}}\left\langle\left(C^{*} \widetilde{\Lambda}_{i}^{*} \widetilde{\Lambda}_{i} C^{\prime}\right)^{\frac{1}{2}} f,\left(C^{*} \Lambda_{i}^{*} \Lambda_{i} C^{\prime}\right)^{\frac{1}{2}} K f\right\rangle-\left\|S_{\rrbracket} f\right\|^{2}=\left\langle K^{*} S_{\rrbracket} f, f\right\rangle-\left\|S_{\rrbracket} f\right\|^{2} \\
& =\left\langle K^{*} S_{\sqrt{J}} f, f\right\rangle-\left\langle S_{\sqrt[J]{ }}^{*} S_{\sqrt{J}} f, f\right\rangle \\
& =\left\langle\left(K-S_{\mathbb{J}}\right)^{*} S_{\rrbracket} f, f\right\rangle \\
& =\left\langle S_{\mathbb{J} c}^{*}\left(K-S_{\sqrt{ } c}\right) f, f\right\rangle \\
& =\left\langle S_{J^{c}}^{*} K f, f\right\rangle-\left\langle S_{J^{c}}^{*} S_{\rrbracket^{c}} f, f\right\rangle \\
& =\left\langle K f, S_{\rrbracket^{c}} f\right\rangle-\left\langle S_{\rrbracket^{c}} f, S_{\rrbracket^{c}} f\right\rangle \\
& =\overline{\left\langle S_{\text {Jc }} f, K f\right\rangle}-\left\|S_{\text {Jc }} f\right\|,
\end{aligned}
$$

and this completes the proof. 
Theorem 3.3. Let $\Lambda$ be a Parseval $C C^{\prime}$-controlled-K-g-frame for $H$. If $\mathbb{J} \subseteq \mathbb{I}$ and $E \subseteq \mathbb{J}^{c}$, then for each $f \in H$

$$
\begin{aligned}
& \left\|\sum_{i \in \mathbb{J} \cup E} C^{*} \Lambda_{i}^{*} \Lambda_{i} C^{\prime} f\right\|^{2}-\left\|\sum_{i \in \mathbb{J}^{c} \backslash E} C^{*} \Lambda_{i}^{*} \Lambda_{i} C^{\prime} f\right\|^{2} \\
= & \left\|\sum_{i \in \mathbb{J}} C^{*} \Lambda_{i}^{*} \Lambda_{i} C^{\prime} f\right\|^{2}-\left\|\sum_{i \in \mathbb{J}^{c}} C^{*} \Lambda_{i}^{*} \Lambda_{i} C^{\prime} f\right\|^{2}+2 \operatorname{Re}\left(\sum_{i \in E}\left\langle\Lambda_{i} C^{\prime} f, \Lambda_{i} C^{*} K K^{*} f\right\rangle\right) .
\end{aligned}
$$

Proof. Let

$$
S_{\Lambda, \mathbb{J}} f:=\sum_{i \in \mathbb{J}} C^{*} \Lambda_{i}^{*} \Lambda_{i} C^{\prime} f .
$$

Therefore, $S_{\Lambda, \mathbb{I}}+S_{\Lambda, \mathbb{I} c}=K K^{*}$. Hence

$$
\begin{aligned}
S_{\Lambda, \mathbb{J}}^{2}-S_{\Lambda, \mathbb{J} c}^{2} & =S_{\Lambda, \mathbb{J}}^{2}-\left(K K^{*}-S_{\Lambda, \mathbb{J}}\right)^{2} \\
& =K K^{*} S_{\Lambda, \mathbb{J}}+S_{\Lambda, \mathbb{J}} K K^{*}-\left(K K^{*}\right)^{2} \\
& =K K^{*} S_{\Lambda, \mathbb{J}}-S_{\Lambda, \mathbb{J} c} K K^{*} .
\end{aligned}
$$

Now, for each $f \in H$ we obtain

$$
\left\|S_{\Lambda, \mathbb{J}}^{2} f\right\|^{2}-\left\|S_{\Lambda, \mathbb{J}^{c}}^{2} f\right\|^{2}=\left\langle K K^{*} S_{\Lambda, \mathbb{J}} f, f\right\rangle-\left\langle S_{\Lambda, \mathbb{J}} K K^{*} f, f\right\rangle .
$$

Consequently, for $\mathbb{J} \cup E$ instead of $\mathbb{J}$ :

$$
\begin{aligned}
& \left\|\sum_{i \in \mathbb{J} \cup E} C^{*} \Lambda_{i}^{*} \Lambda_{i} C^{\prime} f\right\|^{2}-\left\|\sum_{i \in \mathbb{J} \backslash} C^{*} \Lambda_{i}^{*} \Lambda_{i} C^{\prime} f\right\|^{2} \\
= & \sum_{i \in \mathbb{J} \cup E}\left\langle\Lambda_{i} C^{\prime} f, \Lambda_{i} C^{*} K K^{*} f\right\rangle-\sum_{i \in \mathbb{J}^{c} \backslash E} \overline{\left\langle\Lambda_{i} C^{\prime} f, \Lambda_{i} C^{*} K K^{*} f\right\rangle} \\
= & \sum_{i \in \mathbb{J}}\left\langle\Lambda_{i} C^{\prime} f, \Lambda_{i} C^{*} K K^{*} f\right\rangle-\sum_{i \in \mathbb{J}^{c}} \overline{\left\langle\Lambda_{i} C^{\prime} f, \Lambda_{i} C^{*} K K^{*} f\right\rangle} \\
& +2 \operatorname{Re}\left(\sum_{i \in E}\left\langle\Lambda_{i} C^{\prime} f, \Lambda_{i} C^{*} K K^{*} f\right\rangle\right) \\
= & \left\|\sum_{i \in \mathbb{J}} C^{*} \Lambda_{i}^{*} \Lambda_{i} C^{\prime} f\right\|^{2}-\left\|\sum_{i \in \mathbb{J}^{c}} C^{*} \Lambda_{i}^{*} \Lambda_{i} C^{\prime} f\right\|^{2}+2 \operatorname{Re}\left(\sum_{i \in E}\left\langle\Lambda_{i} C^{\prime} f, \Lambda_{i} C^{*} K K^{*} f\right\rangle\right) .
\end{aligned}
$$

Theorem 3.4. Let $\Lambda$ be a Parseval $C C^{\prime}$-controlled-K-g-frame for $H$. If $\mathbb{J} \subseteq \mathbb{I}$, then for each $f \in H$

$$
\begin{aligned}
& \left\|\sum_{i \in \mathbb{J}} C^{*} \Lambda_{i}^{*} \Lambda_{i} C^{\prime} f\right\|^{2}+\operatorname{Re}\left(\sum_{i \in J^{c}}\left\langle\Lambda_{i} C^{\prime} f, \Lambda_{i} C^{*} K K^{*} f\right\rangle\right) \\
= & \left\|\sum_{i \in \mathbb{J}^{c}} C^{*} \Lambda_{i}^{*} \Lambda_{i} C^{\prime} f\right\|^{2}+\operatorname{Re}\left(\sum_{j \in \mathbb{J}}\left\langle\Lambda_{i} C^{\prime} f, \Lambda_{i} C^{*} K K^{*} f\right\rangle\right) \geq \frac{3}{4}\left\|K K^{*} f\right\|^{2} .
\end{aligned}
$$

Proof. By the proof of Theorem 3.3, we have

$$
S_{\Lambda, \mathbb{J}}^{2}-S_{\Lambda, \mathbb{J} c}^{2}=K K^{*} S_{\Lambda, \mathbb{J}}-S_{\Lambda, \mathbb{J} c} K K^{*} .
$$

Therefore

$$
S_{\Lambda, \mathbb{J}}^{2}+S_{\Lambda, \mathbb{I} c}^{2}=2\left(\frac{K K^{*}}{2}-S_{\Lambda, \mathbb{J}}\right)^{2}+\frac{\left(K K^{*}\right)^{2}}{2} \geq \frac{\left(K K^{*}\right)^{2}}{2} .
$$


Thus

$$
\begin{aligned}
K K^{*} S_{\Lambda, \mathbb{J}}+S_{\Lambda, \mathbb{J} c}^{2}+\left(K K^{*} S_{\Lambda, \mathbb{J}}+S_{\Lambda, \mathbb{J c}}^{2}\right)^{*} & =K K^{*} S_{\Lambda, \mathbb{J}}+S_{\Lambda, \mathbb{J} c}^{2}+S_{\Lambda, \mathbb{J}} K K^{*}+S_{\Lambda, \mathbb{J} c}^{2} \\
& =K K^{*}\left(S_{\Lambda, \mathbb{J}}+S_{\Lambda, \mathbb{J}}\right)+S_{\Lambda, \mathbb{J}}^{2}+S_{\Lambda, \mathbb{J} c}^{2} \\
& \geq \frac{3}{2}\left(K K^{*}\right)^{2} .
\end{aligned}
$$

Now, for each $f \in H$ we obtain

$$
\begin{aligned}
& \left\|\sum_{i \in \mathbb{J}} C^{*} \Lambda_{i}^{*} \Lambda_{i} C^{\prime} f\right\|^{2}+\operatorname{Re}\left(\sum_{i \in J^{c}}\left\langle\Lambda_{i} C^{\prime} f, \Lambda_{i} C^{*} K K^{*} f\right\rangle\right) \\
& =\frac{1}{2}\left(\left\langle K K^{*} S_{\Lambda, \mathbb{J}} f, f\right\rangle+\left\langle S_{\Lambda, \mathbb{J}}^{2} f, f\right\rangle+\left\langle f, K K^{*} S_{\Lambda, \mathbb{J}} f\right\rangle+\left\langle f, S_{\Lambda, \mathbb{J} c}^{2} f\right\rangle\right) \\
& \geq \frac{3}{4}\left\|K K^{*} f\right\|^{2} .
\end{aligned}
$$

\section{Perturbation of controlled $K$-g-frames}

Stability of the wavelet and Gabor frames under perturbation is one of the important problems in frame theory. At first this problem was studied by Paley and Wienes for bases and then extended to frames. In recent years many authors extended the concept of perturbation to many kinds of frames such as g-frames, Banach frames, $\mathrm{K}$ and controlled frames $[5,11,12,19,20]$. But the most important results are obtained by Casazza and Christensen in [3]. Here we study the perturbation of $C C^{\prime}$-controlled $K$-g-frames.

Theorem 4.1. Let $\Lambda$ be a $C C^{\prime}$-controlled $K$-g-frame for $H$ with bounds $A_{C C^{\prime}}$ and $B_{C C^{\prime}}$. Assume that $\Theta:=\left\{\Theta_{i} \in \mathcal{B}\left(H, H_{i}\right)\right\}_{i \in \mathbb{I}}$ is a sequence of operators such that for each $f \in H$ and $i \in \mathbb{I}$

$$
\begin{gathered}
\left\|\left(C^{*} \Lambda_{i}^{*} \Lambda_{i} C^{\prime}-C^{*} \Theta_{i}^{*} \Theta_{i} C^{\prime}\right)^{\frac{1}{2}} f\right\| \leq \lambda_{1}\left\|\left(C^{*} \Lambda_{i}^{*} \Lambda_{i} C^{\prime}\right)^{\frac{1}{2}} f\right\|+\lambda_{2}\left\|\left(C^{*} \Theta_{i}^{*} \Theta_{i} C^{\prime}\right)^{\frac{1}{2}} f\right\| \\
+c_{i}\left\|K^{*} f\right\|,
\end{gathered}
$$

where $\left\{c_{i}\right\}_{i \in \mathbb{I}}$ is a sequence of positive numbers such that $\eta:=\sum_{i \in \mathbb{I}} c_{i}^{2}<\infty$ and $0 \leq \lambda_{1}, \lambda_{2}<1$. Then $\Theta$ is a $C C^{\prime}$ controlled $K$-g-frame for $H$ with bounds:

$$
\left(\frac{\left(1-\lambda_{1}\right) \sqrt{A_{C C^{\prime}}}-\eta}{1+\lambda_{2}}\right)^{2}, \quad\left(\frac{\left(1+\lambda_{1}\right) \sqrt{B_{C C^{\prime}}}+\eta\|K\|}{1-\lambda_{2}}\right)^{2} .
$$

Proof. For each $f \in H$ we have

$$
\begin{aligned}
\left\|\left(C^{*} \Theta_{i}^{*} \Theta_{i} C^{\prime}\right)^{\frac{1}{2}} f\right\|= & \left\|\left(C^{*} \Theta_{i}^{*} \Theta_{i} C^{\prime}-C^{*} \Lambda_{i}^{*} \Lambda_{i} C^{\prime}\right)^{\frac{1}{2}} f+\left(C^{*} \Lambda_{i}^{*} \Lambda_{i} C^{\prime}\right)^{\frac{1}{2}} f\right\| \\
\leq & \left\|\left(C^{*} \Theta_{i}^{*} \Theta_{i} C^{\prime}-C^{*} \Lambda_{i}^{*} \Lambda_{i} C^{\prime}\right)^{\frac{1}{2}} f\right\|+\left\|\left(C^{*} \Lambda_{i}^{*} \Lambda_{i} C^{\prime}\right)^{\frac{1}{2}} f\right\| \\
\leq & \lambda_{1}\left\|\left(C^{*} \Lambda_{i}^{*} \Lambda_{i} C^{\prime}\right)^{\frac{1}{2}} f\right\|+\lambda_{2}\left\|\left(C^{*} \Theta_{i}^{*} \Theta_{i} C^{\prime}\right)^{\frac{1}{2}} f\right\| \\
& +c_{i}\left\|K^{*} f\right\|+\left\|\left(C^{*} \Lambda_{i}^{*} \Lambda_{i} C^{\prime}\right)^{\frac{1}{2}} f\right\| .
\end{aligned}
$$

Hence

$$
\left(1-\lambda_{2}\right)\left\|\left(C^{*} \Theta_{i}^{*} \Theta_{i} C^{\prime}\right)^{\frac{1}{2}} f\right\| \leq\left(1+\lambda_{1}\right)\left\|\left(C^{*} \Lambda_{i}^{*} \Lambda_{i} C^{\prime}\right)^{\frac{1}{2}} f\right\|+c_{i}\left\|K^{*} f\right\| .
$$


Since $\Lambda$ is a $C C^{\prime}$-controlled $K$-g-frame, so

$$
\begin{aligned}
\left\|T_{C C^{\prime}}^{*} f\right\|^{2} & =\left\|\left(C^{*} \Lambda_{i}^{*} \Lambda_{i} C^{\prime}\right)^{\frac{1}{2}} f\right\|^{2} \\
& =\sum_{i \in \mathbb{I}}\left\langle\Lambda_{i} C^{\prime} f, \Lambda_{i} C f\right\rangle \\
& \leq B_{C C^{\prime}}\|f\|^{2} .
\end{aligned}
$$

Therefore

$$
\begin{aligned}
\left\|\left(C^{*} \Theta_{i}^{*} \Theta_{i} C^{\prime}\right)^{\frac{1}{2}} f\right\| & \leq \frac{\left(1+\lambda_{1}\right)\left\|\left(C^{*} \Lambda_{i}^{*} \Lambda_{i} C^{\prime}\right)^{\frac{1}{2}} f\right\|+c_{i}\left\|K^{*} f\right\|}{1-\lambda_{2}} \\
& \leq\left(\frac{\left(1+\lambda_{1}\right) \sqrt{B_{C C^{\prime}}}+\eta\|K\|}{1-\lambda_{2}}\right)\|f\| .
\end{aligned}
$$

Now, for the lower bound we get

$$
\begin{aligned}
\left\|\left(C^{*} \Theta_{i}^{*} \Theta_{i} C^{\prime}\right)^{\frac{1}{2}} f\right\|= & \left\|\left(C^{*} \Lambda_{i}^{*} \Lambda_{i} C^{\prime}\right)^{\frac{1}{2}} f-\left(C^{*} \Lambda_{i}^{*} \Lambda_{i} C^{\prime}-C^{*} \Theta_{i}^{*} \Theta_{i} C^{\prime}\right)^{\frac{1}{2}} f\right\| \\
\geq & \left\|\left(C^{*} \Lambda_{i}^{*} \Lambda_{i} C^{\prime}\right)^{\frac{1}{2}} f\right\|-\left\|\left(C^{*} \Lambda_{i}^{*} \Lambda_{i} C^{\prime}-C^{*} \Theta_{i}^{*} \Theta_{i} C^{\prime}\right)^{\frac{1}{2}} f\right\| \\
\geq & \left\|\left(C^{*} \Lambda_{i}^{*} \Lambda_{i} C^{\prime}\right)^{\frac{1}{2}} f\right\|-\lambda_{1}\left\|\left(C^{*} \Lambda_{i}^{*} \Lambda_{i} C^{\prime}\right)^{\frac{1}{2}} f\right\| \\
& \quad-\lambda_{2}\left\|\left(C^{*} \Theta_{i}^{*} \Theta_{i} C^{\prime}\right)^{\frac{1}{2}} f\right\|-c_{i}\left\|K^{*} f\right\| .
\end{aligned}
$$

Therefore

$$
\begin{aligned}
& \left(1+\lambda_{2}\right)\left\|\left(C^{*} \Theta_{i}^{*} \Theta_{i} C^{\prime}\right)^{\frac{1}{2}} f\right\| \\
& \quad \geq\left(1-\lambda_{1}\right)\left\|\left(C^{*} \Lambda_{i}^{*} \Lambda_{i} C^{\prime}\right)^{\frac{1}{2}} f\right\|-c_{i}\left\|K^{*} f\right\|,
\end{aligned}
$$

or

$$
\left\|\left(C^{*} \Theta_{i}^{*} \Theta_{i} C^{\prime}\right)^{\frac{1}{2}} f\right\| \geq \frac{\left(1-\lambda_{1}\right)\left\|\left(C^{*} \Lambda_{i}^{*} \Lambda_{i} C^{\prime}\right)^{\frac{1}{2}} f\right\|-c_{i}\left\|K^{*} f\right\|}{1+\lambda_{2}} .
$$

Since,

$$
\left\|T_{C C^{\prime}}^{*} f\right\|^{2}=\left\|\left(C^{*} \Lambda_{i}^{*} \Lambda_{i} C^{\prime}\right)^{\frac{1}{2}} f\right\|^{2} \geq A_{C C^{\prime}}\left\|K^{*} f\right\|^{2},
$$

thus

$$
\left\|\left(C^{*} \Theta_{i}^{*} \Theta_{i} C^{\prime}\right)^{\frac{1}{2}} f\right\| \geq\left(\frac{\left(1-\lambda_{1}\right) \sqrt{A_{C C^{\prime}}}-\eta}{1+\lambda_{2}}\right)\left\|K^{*} f\right\| .
$$

This completes the proof.

Proposition 4.1. Let $\Lambda$ be a $C C^{\prime}$-controlled $K$-g-frame for $H$ with bounds $A_{C C^{\prime}}$ and $B_{C C^{\prime}}$. Assume that $\Theta:=\left\{\Theta_{i} \in \mathcal{B}\left(H, H_{i}\right)\right\}_{i \in \mathbb{I}}$ is a sequence of operators such that for each $f \in H$ and $i \in \mathbb{I}$

$$
\left\|\left(C^{*} \Lambda_{i}^{*} \Lambda_{i} C^{\prime}-C^{*} \Theta_{i}^{*} \Theta_{i} C^{\prime}\right)^{\frac{1}{2}} f\right\| \leq c_{i}\left\|K^{*} f\right\|,
$$

where $\left\{c_{i}\right\}_{i \in \mathbb{I}}$ is a sequence of positive numbers such that $\eta:=\sum_{i \in \mathbb{I}} c_{i}^{2}<\infty$. Then $\Theta$ is a $C C^{\prime}$-controlled $K$-g-frame for $H$ with bounds:

$$
\left(\sqrt{A_{C C^{\prime}}}-\eta\right)^{2}, \quad\left(\eta\|K\|+\sqrt{B_{C C^{\prime}}}\right)^{2} .
$$


Proof. For each $f \in H$ we have

$$
\begin{aligned}
\left\|\left(C^{*} \Theta_{i}^{*} \Theta_{i} C^{\prime}\right)^{\frac{1}{2}} f\right\| & =\left\|\left(C^{*} \Theta_{i}^{*} \Theta_{i} C^{\prime}-C^{*} \Lambda_{i}^{*} \Lambda_{i} C^{\prime}\right)^{\frac{1}{2}} f+\left(C^{*} \Lambda_{i}^{*} \Lambda_{i} C^{\prime}\right)^{\frac{1}{2}} f\right\| \\
& \leq\left\|\left(C^{*} \Theta_{i}^{*} \Theta_{i} C^{\prime}-C^{*} \Lambda_{i}^{*} \Lambda_{i} C^{\prime}\right)^{\frac{1}{2}} f\right\|+\left\|\left(C^{*} \Lambda_{i}^{*} \Lambda_{i} C^{\prime}\right)^{\frac{1}{2}} f\right\| \\
& \leq\left(\eta\|K\|+\sqrt{B C C^{\prime}}\right)\|f\| .
\end{aligned}
$$

Therefore $\Theta$ is a $C C^{\prime}$-controlled g-Bessel sequence for $H$. On the other hand

$$
\begin{aligned}
\left\|\left(C^{*} \Theta_{i}^{*} \Theta_{i} C^{\prime}\right)^{\frac{1}{2}} f\right\| & \geq\left\|\left(C^{*} \Lambda_{i}^{*} \Lambda_{i} C^{\prime}\right)^{\frac{1}{2}} f\right\|-\left\|\left(C^{*} \Lambda_{i}^{*} \Lambda_{i} C^{\prime}-C^{*} \Theta_{i}^{*} \Theta_{i} C^{\prime}\right)^{\frac{1}{2}} f\right\| \\
& \geq\left(\sqrt{A_{C C^{\prime}}}-\eta\right)\left\|K^{*} f\right\|,
\end{aligned}
$$

and this completes the proof.

\section{Acknowledgements}

The authors would like to express their gratitude to the reviewers for helpful comments.

\section{References}

[1] P. Balazs, J. P. Antoine and A. Grybos, Weighted and controlled frames: mutual relationship and first numerical properties, Int. J. Wavelets Multiresolut. Inf. Process. 8, (2010), no. 1, 109-132.

[2] I. Bogdanova, P. Vandergheynst, J. .P. Antoine, L. Jacques and M. Morvidone, Stereographic wavelet frames on the sphere, Appl. Comput. Harmon. Anal. 19, (2005), no.2, 223-252.

[3] P. G. Casazza and O. Christensen, Perturbation of Operators and Application to Frame Theory, J. Fourier Anal. Appl. 3, (1997), no. 5, 543-557.

[4] O. Christensen, An Introduction to Frames and Riesz Bases, Birkhäuser, Boston, 2002.

[5] O. Christensen, and CH. Heil, Perturbation of Banach frames and atomic decomposition, Math. Nachr. 185, (1997), 33-47.

[6] R. G. Douglas, On majorization, Factorization and Range Inclusion of Operators on Hilbert Spaces, Proc Amer. Math. Soc. 17, (1996), no. 2, 413-415.

[7] R. J. Duffin, and A. C. Schaeffer, A class of nonharmonic Fourier series, Trans. Amer. Math. Soc. 72, (1952), no. 2, 341-366.

[8] M. Forughi, E. Osgooei, A. Rahimi and M. Javahernia, An appropriate representation space for controlled G-frames, arXiv:1905.08962 [math.FA].

[9] L. Găvruţa, Frames for Operators, Appl. Comput. Harmon. Anal. 32, (2012), no. 1, 139-144.

[10] D. Hua and Y. Huang, Controlled $K$-g-frames in Hilbert spaces, Results Math. 72, (2017), no. 3, 1227-1238.

[11] D. Li and J. Leng, Generalized frames for controlled operators in Hilbert spaces, Ann. Funct. Anal. 10, (2019), no. 4, 537-552.

[12] K. Musazadeh and H. Khandani, Some results on controlled frames in Hilbert spaces, Acta Math. Sci. 36, (2016), no. 3, 655-665.

[13] A. Najati, M. H. Faroughi and A. Rahimi, G-frames and stability of g-frames in Hilbert spaces, Methods Funct. Anal. Topology. 14, (2008), no. 3, 271-286.

[14] M. Nouri, A. Rahimi and S. Najafzadeh, Controlled $K$-frames in Hilbert spaces, $J$. Ramanajan Math. Soc. 4, (2015), no.2, 39-50. 
[15] E. Osgooei, A. Rahimi, Cross-Gram matrix associated to two sequences in Hilbert spaces, Iran. J. Sci. Technol. Trans. A Sci. 43, (2019), no. 4, 1755-1760.

[16] E. Osgooei, and A. Rahimi, Gram matrix associated to controlled frames, Int. J. Wavelets Multiresolut. Inf. Process. 16, (2018), no. 5, 1-15.

[17] A. Rahimi and A. Fereydooni, Controlled G-frames and their G-multipliers in Hilbert spaces, An. St. Univ. Ovidius Constanta 21, (2013), no. 2, 223-236.

[18] W. Sun, G-Frames and G-Riesz bases, J. Math. Anal. Appl. 322, (2006), 437-452.

[19] W. Sun, Stability of G-frames, J. Math. Anal. Appl. 326, (2007), 858-868.

[20] X. Xiao, Y. Zhu, and L. Găvruţa, Some properties of $K$-frames in Hilbert spaces, Results Math. 63, (2013), ni. 3-4, 1243-1255.

\section{Maryam Forughi}

Department of Mathematics, Shabestar Branch, Islamic Azad University, Shabestar, Iran.

E-mail address: Maryam.forughi@yahoo.com

Elnaz Osgooei

Faculty of Science, Urmia University of Technology, Urmia, Iran.

E-mail address: e.osgooei@uut.ac.ir

Asghar Rahimi

Department of Mathematics, University of Maragheh, Maragheh, Iran.

E-mail address: rahimi@maragheh.ac.ir

Mojgan Javahernia Iran.

Department of Mathematics, Shabestar Branch, Islamic Azad University, Shabestar,

E-mail address: javahernia_math@yahoo.com

Received: May 22, 2019; Revised: November 15, 2019; Accepted: January 28, 2020 